\title{
Three-Body and One-Body Channels of the Auger Core-Valence-Valence decay: Simplified Approach
}

\author{
Andrea Marini and Michele Cini \\ Istituto Nazionale di Fisica della Materia, Dipartimento di Fisica, \\ Universita' di Roma Tor Vergata, Via della Ricerca Scientifica, 1-00133 \\ Roma, Italy
}

\begin{abstract}
We propose a computationally simple model of Auger and APECS line shapes from open-band solids. Part of the intensity comes from the decay of unscreened coreholes and is obtained by the two-body Green's function $G_{\omega}^{(2)}$, as in the case of filled bands. The rest of the intensity arises from screened core-holes and is derived using a variational description of the relaxed ground state; this involves the two-holesone-electron propagator $G_{\omega}$, which also contains one-hole contributions. For many transition metals, the two-hole Green's function $G_{\omega}^{(2)}$ can be well described by the Ladder Approximation, but the three-body Green's function poses serious further problems. To calculate $G_{\omega}$, treating electrons and holes on equal footing, we propose a practical approach to sum the series to all orders. We achieve that by formally rewriting the problem in terms of a fictitious three-body interaction. Our method grants non-negative densities of states, explains the apparent negative- $U$ behavior of the spectra of early transition metals and interpolates well between weak and strong coupling, as we demonstrate by test model calculations.
\end{abstract}

PACS Numbers: 79.20.Fv; 79.60.-i

Keywords: Electron impact: Auger emission; Photoemission and photoelectron spectra 


\section{Introduction}

The Core-Valence-Valence (CVV) Auger Spectra of solids with closed valence bands are well described [1] in terms of the two-holes Green's function $G_{\omega}^{(2)}$ by the Cini [2, 3] and Sawatzky [4] (CS) model.

The CS model allows to understand the phenomenology involving band-like, atomic-like and intermediate situations in terms of the $U / W$ ratio of the on-site repulsion $U$ to the band width $W$. For low $U / W$, the line shape is close to the self-convolution on the local one-hole density of states; with increasing $U / W$, the shape is distorted until, for a critical value of the ratio, two-hole resonances appear. They correspond to poles of $G_{\omega}^{(2)}$, as calculated from Anderson, Hubbard or related models. Atomic-like peaks and band-like structures are often observed [1] in the same spectrum. For high $U / W$ (atomic-like case) the Auger line shapes are so close to the free-atom spectra that they are labeled by LSJ terms and levels. Detailed studies of noble transition metals, like $\mathrm{Au}$ [5] and $\mathrm{Ag}$ [6] led to a very good agreement between theory and experiment, and also allowed the direct observation of off-site interaction effects [7]. A similar success was achieved for covalently bonded solids [8]. In all cases the diagrammatic expansion of $G_{\omega}^{(2)}$ is just a ladder of successive interactions between the holes without self-energy terms or vertex corrections.

For open valence bands, the theory is much more complicated, and it is known from experiment that no such narrow atomic-like peaks exist any more. However for almost completely filled bands, when the number of holes per quantum state $n_{h} \ll 1$, remnants of the atomic multiplet structure are still observed and the closed-band theory can be extrapolated [9]. To a first approximation, one can assume that the valence electrons remain frozen during the core ionisation, and in the initial state of the Auger decay the valence configuration is the same as in the ground state. The intra-band shake-up effects are $O\left(n_{h}\right)$ and can be accounted for by convolving with an asymmetric Doniach-Šunjić [10] line shape. In this way, the CVV spectra are still described in terms of the two-hole Green's function $G_{\omega}^{(2)}$, computed in the absence of the core hole. Using Galitzkii's Low Density Approximation [11] (LDA) the dominant diagrams of the perturbation expansion of the $G_{\omega}^{(2)}$ are just the same ladder diagrams which provide the exact solution for $n_{h} \rightarrow 0$. This gives a satisfactory explanation of the Auger spectra for $n_{h} \approx 0.1$, which includes interesting cases like $\mathrm{Ni}$ and $\mathrm{Pd}$ [12], even in finely dispersed form [13]. To extend the LDA to larger $n_{h}$, a self-consistent version of the Low Density Approximation that involves using dressed propagators in the ladder series was naturally suggested [14. However an unexpected result cames from cluster studies 15 which clearly demonstrated that the ladder approximation with bare propagators is superior and is a good approximation to $G_{\omega}^{(2)}$ for a wide $n_{h}$ range. This is the Bare Ladder Approximation (BLA). This approach has been useful to interpret the line shape of Graphite [16].

The next turn came from experiments on early $3 d$ transition metals, like $\mathrm{Ti}$ and $\mathrm{Sc}$ [17], that could not be interpreted by the above theory. The maximum of the line shape was shifted by the interaction to lower binding energy, which is the contrary of what happens in closed band materials. Qualitatively the CS model could work if one admitted that $U<0$, and such an explanation has actually been proposed [18.

However, no other evidence of $U<0$ was found; rather, it became clear that for almost empty bands one must formulate a new theory which is no simple extrapolation of the closed- 
band approach. Sarma [19] first suggested that the Auger line shape of Ti looks like some linear combination of the one-electron density of states and its convolution. Using this hint, and a general formulation of the Auger decay by Gunnarsson and Schönhammer [20], a simple explanation of the apparent negative- $U$ behavior was found [21]. In this theory, the Auger line shape has two main contributions, that we call unrelaxed and relaxed, respectively. The unrelaxed contribution is obtained assuming that the Auger decay occurs while the conduction electrons are in their ground state $|\psi\rangle$ in the absence of the core hole. The density of states which shows up in this contribution is obtained by $G_{\omega}^{(2)}$, like in closed-band systems. However, this is only a part of the story, and in many ways the easy part, because the screening electronic cloud that surrounds the core-hole can partecipate in the Auger decay. The ground state $|\phi\rangle$ in the presence of the core hole also enters the description. The relaxed contribution is computed with $|\phi\rangle$ as the initial state of the Auger decay. We summarise in the Appendix A the argument wich leads us to express the relaxed contribution in terms of a three-body (the Auger holes and the screening electron) density of states $D$; this is obtained from the Fourier transform of the $t>0$ part of the Green's function

$$
G\left(\alpha_{l} \beta_{l} \gamma_{l}, \gamma_{l}^{\prime} \beta_{l}^{\prime} \alpha_{l}^{\prime} ; t\right)=(-i)^{3}\left\langle\psi\left|T\left\{a_{\alpha_{l}}^{\dagger}(t) a_{\beta_{l}}^{\dagger}(t) a_{\gamma_{l}}(t) a_{\gamma_{l}^{\prime}}^{\dagger} a_{\beta_{l}^{\prime}} a_{\alpha_{l}^{\prime}}\right\}\right| \psi\right\rangle .
$$

Here, operators are in the Heisenberg picture and we introduce a special notation $\alpha_{l}, \beta_{l} \ldots$ to indicate the set of quantum numbers of the local valence spin-orbitals belonging to the atom where the Auger decay occurs (the Auger site, for short). Experimentally, one can single out the relaxed contribution of (1.1) by properly fixing the photoelectron energy in an AugerPhotoelectron Coincidence Spectroscopy (APECS) experiment, 22, 23, 24, 25] where the Auger electron is detected in coincidence with the photoelectron reponsible of the core hole creation. Fixing the photoelectron energy, the Auger electron measured in coincidence comes from the decay of a few dominant intermediate states in the presence of the core hole [26].

Since (1.1) is hard to calculate and even much harder than of $G_{\omega}^{(2)}$, we propose a simple approach in the spirit of the BLA. If we wish to design an affordable scheme in this difficult problem, we must be prepared to adopt a series of approximations: Cini model, Ladder Approximation and a new one, which we call the Core Approximation. Section 2 is devoted to the fomulation of the new scheme. The degree of validity of our approach will be investigated by comparison with exact results from cluster calculations in Section 3 .

\section{Perturbation expansion of the two and three-body Green's functions}

In the Cini model, we express the Auger line shape in terms of local Green's functions, like the one of Equation (1.1); moreover, we calculate them by considering only the local scattering at the Auger site, since in this way we drastically simplify the algebra, and the line shape is little influenced by scattering at the other sites [27]. Many Auger line shape calculations in solids have been performed in this way.

The local interaction approach has been extended successfully to open bands [12, 13. In the present work we want to study its application to more general $n_{h}$. This should allow to 
extend the analysis to several transition metals, giving at least a qualitative understanding of their spectra, which is currently a difficult task.

In order to properly evaluate the results one should bear in mind that currently even for $G_{\omega}^{(2)}$ we have reliable recipes only for $n_{h}$ less than $\approx 0.25$. This problem involves one more body and highly excited states of interacting systems; consequently even the main features of the solution are quite an unsettled question.

\subsection{Ladder Approximation to the two-hole propagator}

The time-dependent Green's function $G_{t}^{(2)}$ allows the usual perturbation expansion, in terms of time-ordered products of interaction-representation operators, namely:

$$
G^{(2)}\left(\alpha_{l} \beta_{l}, \beta_{l}^{\prime} \alpha_{l}^{\prime} ; t\right)=\sum_{n}(-i)^{n} \int_{-\infty}^{\infty} d t_{1} \ldots \int_{-\infty}^{\infty} d t_{n}\left\langle T\left\{a_{\alpha_{l}}^{\dagger}(t) a_{\beta_{l}}^{\dagger}(t) H_{U}\left(t_{1}\right) \ldots H_{U}\left(t_{n}\right) a_{\beta_{l}^{\prime}} a_{\alpha_{l}^{\prime}}\right\}\right\rangle_{c}
$$

with the average taken over the non interacting ground state $\left|\psi_{0}\right\rangle$ and the sum restricted to the topologically inequivalent, connected diagrams. Ordering the spin-orbitals in an arbitrary way, we may write the Coloumb valence-valence interaction between those of the Auger site in the form

$$
H_{U}=\sum_{\mu_{l}<\nu_{l}, \rho_{l}<\tau_{l}} U_{\mu_{l} \nu_{l} \rho_{l} \tau_{l}} a_{\mu_{l}}^{\dagger} a_{\nu_{l}}^{\dagger} a_{\tau_{l}} a_{\rho_{l}}
$$

The diagrammatic method develops Equation (2.1) in terms of local non-interacting time ordered one-body propagator $S_{0}\left(\alpha_{l}, \beta_{l} ; t\right)$

$$
S_{0}\left(\alpha_{l}, \beta_{l} ; t\right)=S_{0}^{h}\left(\alpha_{l}, \beta_{l} ; t\right)-S_{0}^{e}\left(\beta_{l}, \alpha_{l} ;-t\right),
$$

where

$$
\begin{aligned}
S_{0}^{h}\left(\alpha_{l}, \beta_{l} ; t\right) & =-i \theta(t)\left\langle a_{\alpha_{l}}^{\dagger}(t) a_{\beta_{l}}\right\rangle, \\
S_{0}^{e}\left(\beta_{l}, \alpha_{l} ;-t\right) & =-i \theta(-t)\left\langle a_{\beta_{l}} a_{\alpha_{l}}^{\dagger}(t)\right\rangle .
\end{aligned}
$$

here the average is taken over the non-interacting ground state $\left|\psi_{0}\right\rangle$ with energy $E_{0}$. For $n_{h}<0.25$ and a range of $U / W$, a good start is provided by the BLA. In the BLA, one selects the series of ladder diagrams which are free of self-energy insertions and vertex corrections. This is equivalent to the approximate factorisation

$$
\begin{aligned}
\left\langle T\left\{a_{\alpha_{l}}^{\dagger}(t) a_{\beta_{l}}^{\dagger}(t) H_{U}\left(t_{1}\right) \ldots H_{U}\left(t_{n}\right) a_{\beta_{l}^{\prime}} a_{\alpha_{l}^{\prime}}\right\}\right\rangle_{c} \approx \sum_{\mu_{l}<\nu_{l}, \rho_{l}<\tau_{l}} U_{\mu_{l} \nu_{l} \rho_{l} \tau_{l}} \\
\quad\left[\left\langle T\left\{a_{\alpha_{l}}^{\dagger}(t) a_{\beta_{l}}^{\dagger}(t) a_{\tau_{l}}\left(t_{1}\right) a_{\rho_{l}}\left(t_{1}\right)\right\}\right\rangle\left\langle T\left\{a_{\mu_{l}}^{\dagger}\left(t_{1}\right) a_{\nu_{l}}^{\dagger}\left(t_{1}\right) H_{U}\left(t_{2}\right) \ldots H_{U}\left(t_{n}\right) a_{\beta_{l}^{\prime}} a_{\alpha_{l}^{\prime}}\right\}\right\rangle_{c}\right] .
\end{aligned}
$$

Since

$$
g\left(\alpha_{l} \beta_{l}, \tau_{l} \rho_{l} ; t-t_{1}\right)=\left\langle T\left\{a_{\alpha_{l}}^{\dagger}(t) a_{\beta_{l}}^{\dagger}(t) a_{\tau_{l}}\left(t_{1}\right) a_{\rho_{l}}\left(t_{1}\right)\right\}\right\rangle
$$


is the non-interacting Green's function, the BLA leads to

$$
\begin{aligned}
G^{(2)}\left(\alpha_{l} \beta_{l}, \beta_{l}^{\prime} \alpha_{l}^{\prime} ; t\right)= & g\left(\alpha_{l} \beta_{l}, \beta_{l}^{\prime} \alpha_{l}^{\prime} ; t\right) \\
& (-i) \sum_{\mu_{l}<\nu_{l}, \rho_{l}<\tau_{l}} U_{\mu_{l} \nu_{l} \rho_{l} \tau_{l}} \int_{-\infty}^{\infty} d t_{1} g\left(\alpha_{l} \beta_{l}, \tau_{l} \rho_{l} ; t-t_{1}\right) G^{(2)}\left(\mu_{l} \nu_{l}, \beta_{l}^{\prime} \alpha_{l}^{\prime} ; t_{1}\right) .
\end{aligned}
$$

Going to frequency space, this becomes a linear algebraic system. Equation (2.8) is called Bare Ladder Approximation because it uses undressed single particle propagators. It is the exact solution for $n_{h}=0$, and remains a good approximation for a useful range of $n_{h}$. It is simply equivalent to keeping only those diagrams that remain in the closed-band limit. For closed bands, the one-body propagators of Equation (2.3) reduce to the $S_{0}^{h}$ part; therefore, lines that start as hole lines never go back in time and remain hole lines thoughout the diagrams. So, the order of times $t, t_{1}, t_{2}, \ldots, 0$ remains fixed (decreasing) despite the presence of the time-ordering operator $\mathrm{T}$.

The convolution form of Equation (2.8) has furter important consequences. Mathematically, it is a Dyson equation in which the $U$ matrix is an instantaneous self-energy. Therefore, it grants

the Herglotz property: for any interaction strenght, $G^{(2)}$ generates non-negative densities of states. The Herglotz property is a basic requirement for a sensible approximation, yet it is not easily obtained by diagrammatic approaches. Its achievement is one of the most interesting features of this simple approximation.

\subsection{Ladder Approximation to the three-body propagator}

The Green's function (1.1) yields the expansion

$$
\begin{aligned}
G\left(\alpha_{l} \beta_{l} \gamma_{l}, \gamma_{l}^{\prime} \beta_{l}^{\prime} \alpha_{l}^{\prime} ; t\right)=\sum_{n}(-1)^{n}(-i)^{3 n+3} \int_{-\infty}^{\infty} d t_{1} \ldots \int_{-\infty}^{\infty} d t_{n} \\
\left\langle T\left\{a_{\alpha_{l}}^{\dagger}(t) a_{\beta_{l}}^{\dagger}(t) a_{\gamma_{l}}(t) H_{U}\left(t_{1}\right) \ldots H_{U}\left(t_{n}\right) a_{\gamma_{l}^{\prime}}^{\dagger} a_{\beta_{l}^{\prime}} a_{\alpha_{l}^{\prime}}\right\}\right\rangle_{c} .
\end{aligned}
$$

This describes the propagation of two-holes and one electron in the final state, or, if the electron and one hole annihilate, a one-body propagation results (see Section 2.5). In proposing an approximation to Equation (2.9), we may proceed by analogy with $G_{\omega}^{2}$. For $G_{\omega}$ it is natural to propose a bare ladder approximation in which the lines that start as electron (hole) lines never go back in time and remain electron (hole) lines throughout the diagrams; such an approximation should have essentialy the same physical contents as in the previous case and work properly in a wide range of $U$ and fillings. However, the series cannot be summed easily like in Equation (2.8), because with three bodies involved we meet an extra difficulty. For instance, let $H_{U}\left(t_{i}\right)$ produce an interaction between the two holes in a given term of the expansion; then, the electron line overtakes time $t_{i}$; therefore the diagram does not yield a convolution of a function of $t-t_{i}$ times a function of $t_{i}$. This undesirable feature can be removed by using the identities

$$
S_{0}^{h}\left(\alpha_{l}, \beta_{l} ; t\right)=i \sum_{\gamma} S_{0}^{h}\left(\alpha_{l}, \gamma ; t-t^{\prime}\right) S_{0}^{h}\left(\gamma, \beta_{l} ; t^{\prime}\right)
$$



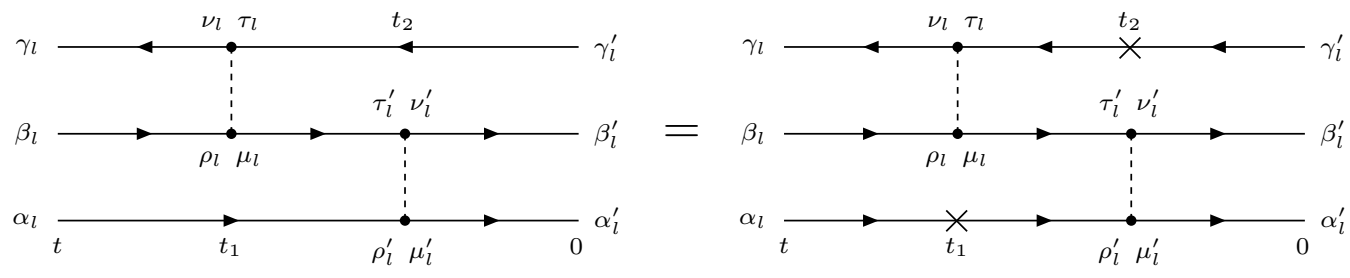

where

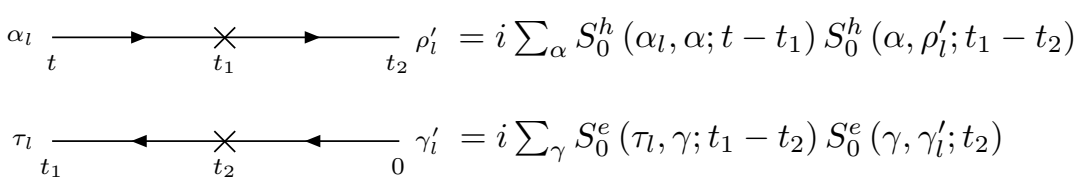

Figure 1: Second order contribution to the three-body Green's function. Using Equations (2.10) we cast it in the form of a product of three "blocks". These are easly dealt with by a Fourier transform.

and

$$
S_{0}^{e}\left(\alpha_{l}, \beta_{l} ; t\right)=i \sum_{\gamma} S_{0}^{e}\left(\alpha_{l}, \gamma ; t-t^{\prime}\right) S_{0}^{e}\left(\gamma, \beta_{l} ; t^{\prime}\right)
$$

where the summations run over all the complete set of spin-orbitals. These identities are derived in Appendix B. Here we note that in the limit $t=t^{\prime} \rightarrow 0^{+}$, we get

$$
S_{0}^{h}\left(\alpha_{l}, \beta_{l} ; t\right) \rightarrow-i \sum_{\gamma}\left\langle a_{\alpha}^{\dagger} a_{\gamma}\right\rangle\left\langle a_{\gamma}^{\dagger} a_{\beta}\right\rangle
$$

and

$$
S_{0}^{e}\left(\alpha_{l}, \beta_{l} ; t\right) \rightarrow i \sum_{\gamma}\left\langle a_{\alpha_{l}} a_{\gamma}^{\dagger}\right\rangle\left\langle a_{\gamma} a_{\beta_{l}}^{\dagger}\right\rangle
$$

Since the ground state is not the hole vacuum, $\left\langle a_{\gamma} a_{\beta}^{\dagger}\right\rangle \neq \delta(\gamma, \beta)$; so the correct limits are obtained only thanks to the completeness of the $\gamma$ set.

To see the use of Equations (2.10), consider for instance the application to one of the second-order contributions to (2.9). Using the standard diagrammatic rules, the l.h.s. of the uppermost Equation in Figure (11) reads (understanding a sum over repeated indices):

$$
\begin{aligned}
& G_{2}\left(\alpha_{l} \beta_{l} \gamma_{l}, \gamma_{l}^{\prime} \beta_{l}^{\prime} \alpha_{l}^{\prime} ; t\right)=-U_{\mu_{l} \nu_{l} \rho_{l} \tau_{l}} U_{\mu_{l}^{\prime} \nu_{l}^{\prime} \rho_{l}^{\prime} \tau_{l}^{\prime}}(-i)^{2} \iint_{-\infty}^{\infty} d t_{1} d t_{2} \\
& {\left[S_{0}^{e}\left(\gamma_{l}, \nu_{l} ; t-t_{1}\right) S_{0}^{h}\left(\beta_{l}, \rho_{l} ; t-t_{1}\right) S_{0}^{h}\left(\alpha_{l}, \rho_{l}^{\prime} ; t-t_{2}\right)\right.} \\
&\left.S_{0}^{h}\left(\mu_{l}, \tau_{l}^{\prime} ; t_{1}-t_{2}\right) S_{0}^{e}\left(\tau_{l}, \gamma_{l}^{\prime} ; t_{2}\right) S_{0}^{h}\left(\nu_{l}^{\prime}, \beta_{l}^{\prime} ; t_{2}\right) S_{0}^{h}\left(\mu_{l}^{\prime}, \alpha_{l}^{\prime} ; t_{2}\right)\right],
\end{aligned}
$$


Using the identities (2.10), one casts Equation (2.12) in the convolution form

$$
\begin{aligned}
& G_{2}\left(\alpha_{l} \beta_{l} \gamma_{l}, \gamma_{l}^{\prime} \beta_{l}^{\prime} \alpha_{l}^{\prime} ; t\right)=-U_{\mu_{l} \nu_{l} \rho_{l} \tau_{l}} U_{\mu_{l}^{\prime} \nu_{l}^{\prime} \rho_{l}^{\prime} \tau_{l}^{\prime}} \\
& \quad \sum_{\alpha, \gamma} \iint_{-\infty}^{\infty} d t_{1} d t_{2}\left[G_{0}\left(\alpha_{l} \beta_{l} \gamma_{l}, \nu_{l} \rho_{l} \alpha ; t-t_{1}\right) G_{0}\left(\alpha \mu_{l} \tau_{l}, \gamma \tau_{l}^{\prime} \rho_{l}^{\prime} ; t_{1}-t_{2}\right) G_{0}\left(\mu_{l}^{\prime} \nu_{l}^{\prime} \gamma, \gamma_{l}^{\prime} \beta_{l}^{\prime} \alpha_{l}^{\prime} ; t_{2}\right)\right] .
\end{aligned}
$$

where

$$
G_{0}\left(\alpha \beta \gamma, \gamma^{\prime} \beta^{\prime} \alpha^{\prime} ; t\right)=S_{0}^{e}\left(\gamma, \gamma^{\prime} ; t\right) S_{0}^{h}\left(\beta, \beta^{\prime} ; t\right) S_{0}^{h}\left(\alpha, \alpha^{\prime} ; t\right)
$$

This is the expression, that we represent pictorally as the r.h.s. of the uppermost Equation in Figure (11). In this way, introducing a fictitious $\times$ interaction vertex, along with the true interaction vertex (dot), we obtain a factorisation similar to Equation (2.6) and the diagram is cast in the convolution form. This useful property extends to all the diagrams of the bare-ladder approximation.

\subsection{Core-Approximation}

We achieved Equation (2.13) in the form of a convolution, which is a big simplification. However, the infinite summations (one for each $\times$ interaction) are a high price to pay for that. They arise because Equations (2.10) imply a summation over the complete set of $\gamma$. On the other hand, since we use a local $H_{U}$, the dot interaction involves only local matrix elements between spin-orbitals; so, we are only interested in the local elements $S^{h, e}\left(\alpha_{l}, \beta_{l} ; t\right)$. Physically, we may expect that only the sites which are closest to the Auger site give an important contribution to the summations, and we can actually work with a limited set to express the local $S^{h, e}\left(\alpha_{l}, \beta_{l} ; t\right)$ elements. Larger sets will lead to more precise results, at the cost of more computation. Here, we wish to explore the simplest approximation, by drastically limiting the $\gamma$ summation to the local states $\gamma_{l}$. We have observed above that summing over the complete $\gamma$ set is necessary to get the correct zero-time limit. In turn, this is essential to preserve normalisation. If we simply replace $\gamma$ with $\gamma_{l}$ in Equations (2.10) this condition is violated (see Equations (2.11)); therefore we introduce a set of functions $R^{ \pm}\left(\alpha_{l}, \beta_{l} ; t\right)$ which approximately factor the propagator in analogy with (2.10) according to the ansatz

$$
(-i)\left\langle a_{\alpha_{l}}^{\dagger}(t) a_{\beta_{l}}\right\rangle \approx \sum_{\gamma_{l}} R^{+}\left(\alpha_{l}, \gamma_{l} ; t-t^{\prime}\right)\left\langle a_{\gamma_{l}}^{\dagger}\left(t^{\prime}\right) a_{\beta_{l}}\right\rangle,
$$

and

$$
(-i)\left\langle a_{\alpha_{l}}(t) a_{\beta_{l}}^{\dagger}\right\rangle \approx \sum_{\gamma_{l}} R^{-}\left(\alpha_{l}, \gamma_{l} ; t-t^{\prime}\right)\left\langle a_{\gamma_{l}}\left(t^{\prime}\right) a_{\beta_{l}}^{\dagger}\right\rangle
$$

where $t^{\prime}$ is any time intermediate between 0 and $t$; the $R^{( \pm)}$functions are computed for any $\mathrm{t}$ by solving the system

$$
(-i)\left\langle a_{\alpha_{l}}^{\dagger}(t) a_{\beta_{l}}\right\rangle=\sum_{\gamma_{l}} R^{+}\left(\alpha_{l}, \gamma_{l} ; t\right)\left\langle a_{\gamma_{l}}^{\dagger} a_{\beta_{l}}\right\rangle
$$


and

$$
(-i)\left\langle a_{\alpha_{l}}(t) a_{\beta_{l}}^{\dagger}\right\rangle=\sum_{\gamma_{l}} R^{-}\left(\alpha_{l}, \gamma_{l} ; t\right)\left\langle a_{\gamma_{l}} a_{\beta_{l}}^{\dagger}\right\rangle
$$

The system must be identically satisfied for any $\mathrm{t}$ and in particular the correct $t \rightarrow 0^{+}$limit is granted.

Equations (2.16) are correct in the limit of core states, when $S^{h, e}\left(\alpha_{l}, \beta_{l} ; t\right)$ is diagonal in its indices and coincides with $R^{ \pm}$; therefore we call this the Core Approximation (CA). The ansatz is also correct in the strong coupling case, when localised two-hole resonances develop. This is appealing, since the strong coupling case is the hard one, while at weak coupling practically every reasonable approach yields similar results. Thus, we regard the ansatz (2.15) as a physically motivated aproximation, which must be tested against exact results for its validation.

\subsection{Summing the Three-body ladder}

Working out the Core Approximation (CA) like in the example (2.13) one can compute all kinds of ladder diagrams, to all orders. The partial sum of the series (2.9) that one obtains in this way will be referred to as Core-Ladder-Approximation (CLA). From now on only local indices appear so we shall dispense ourselves from showing this explicitly. In the exact expansion (2.9), with the local $H_{U}$ denoting the interaction Hamiltonian (2.2) between fermions at the Auger site, the outgoing holes at time $\mathrm{t}$ are labeled $\alpha$ and $\beta$ (creation operators) and the outgoing electron $\gamma$ (annihilation operator). In the CA for the $\mathrm{n}$-th term of (2.9),

$$
\begin{gathered}
G_{n}\left(\alpha \beta \gamma, \gamma^{\prime} \beta^{\prime} \alpha^{\prime} ; t\right)=(-1)^{n}(-i)^{3 n+3} \int_{-\infty}^{\infty} d t_{1} \int_{-\infty}^{\infty} d t_{2} \ldots \int_{-\infty}^{\infty} d t_{n} \sum_{\mu<\nu, \rho<\tau} U_{\mu \nu \rho \tau} \\
\left\langle T\left\{a_{\alpha}^{\dagger}(t) a_{\beta}^{\dagger}(t) a_{\gamma}(t) a_{\mu}^{\dagger}\left(t_{1}\right) a_{\nu}^{\dagger}\left(t_{1}\right) a_{\tau}\left(t_{1}\right) a_{\rho}\left(t_{1}\right) H_{U}\left(t_{2}\right) \ldots H_{U}\left(t_{n}\right) a_{\gamma^{\prime}}^{\dagger} a_{\beta^{\prime}} a_{\alpha^{\prime}}\right\}\right\rangle_{c},
\end{gathered}
$$

we consider the times ordered with $t \geq t_{1} \geq \ldots \geq 0$; let us first analyse the contribution which arises when $H_{U}\left(t_{1}\right)$ describes hole-hole scattering. In the expansion of the T-Product of Equation (2.17) by Wick's theorem we contract the $\alpha$ and $\beta$ creation operators of the outgoing holes with the annihilation operators at time $t_{1}$, obtaining among other terms:

$$
\left\langle T\left\{a_{\alpha}^{\dagger}(t) a_{\rho}\left(t_{1}\right)\right\}\right\rangle\left\langle T\left\{a_{\beta}^{\dagger}(t) a_{\tau}\left(t_{1}\right)\right\}\right\rangle\left\langle T\left\{a_{\gamma}(t) a_{\mu}^{\dagger}\left(t_{1}\right) a_{\nu}^{\dagger}\left(t_{1}\right) H_{U}\left(t_{2}\right) \ldots a_{\gamma^{\prime}}^{\dagger} a_{\beta^{\prime}} a_{\alpha^{\prime}}\right\}\right\rangle_{c},
$$

This, however cannot yet be factored in the convolution form because the last term of Equation (2.18) depends on $t$. In the ladder approximation, the annihilation operator $a_{\gamma}(t)$ can be contracted with any creation operator at whatever time $t_{2}, \ldots, t_{n}$ or 0 . Using the CA we can insert $\mathrm{a} \times$ vertex at time $t_{1}$ on the $\gamma$ electronic line (like in Figure (11)); namely

$$
\begin{aligned}
\left\langle T\left\{a_{\alpha}^{\dagger}(t) a_{\rho}\left(t_{1}\right)\right\}\right\rangle\left\langle T\left\{a_{\beta}^{\dagger}(t) a_{\tau}\left(t_{1}\right)\right\}\right\rangle\left\langle T\left\{a_{\gamma}(t) a_{\mu}^{\dagger}\left(t_{1}\right) a_{\nu}^{\dagger}\left(t_{1}\right) H_{U}\left(t_{2}\right) \ldots a_{\gamma^{\prime}}^{\dagger} a_{\beta^{\prime}} a_{\alpha^{\prime}}\right\}\right\rangle_{c}= \\
\left\langle T\left\{a_{\alpha}^{\dagger}(t) a_{\rho}\left(t_{1}\right)\right\}\right\rangle\left\langle T\left\{a_{\beta}^{\dagger}(t) a_{\tau}\left(t_{1}\right)\right\}\right\rangle \sum_{\xi} i R^{-}\left(\gamma, \xi ; t-t_{1}\right) \\
\left\langle T\left\{a_{\xi}\left(t_{1}\right) a_{\mu}^{\dagger}\left(t_{1}\right) a_{\nu}^{\dagger}\left(t_{1}\right) H_{U}\left(t_{2}\right) \ldots a_{\gamma^{\prime}}^{\dagger} a_{\beta^{\prime}} a_{\alpha^{\prime}}\right\}\right\rangle_{c} .
\end{aligned}
$$




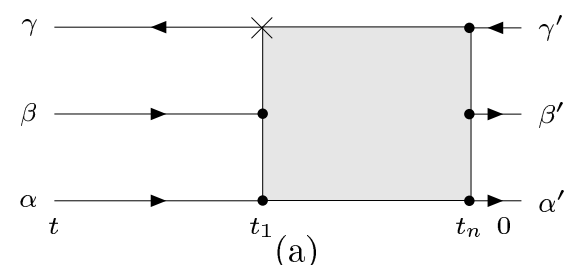

(a)

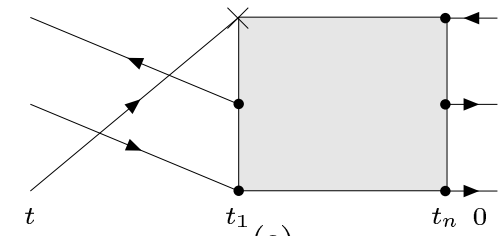

(c)

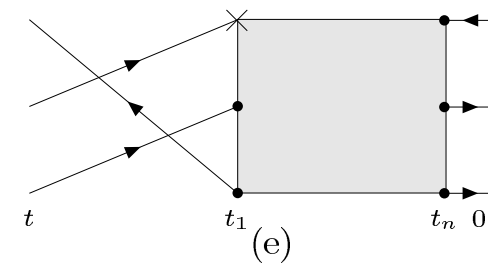

(e)

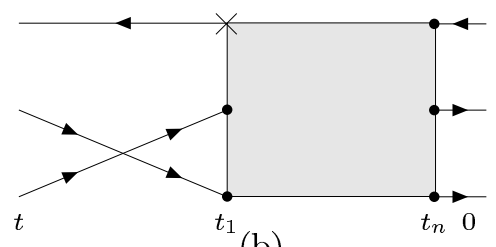

(b)

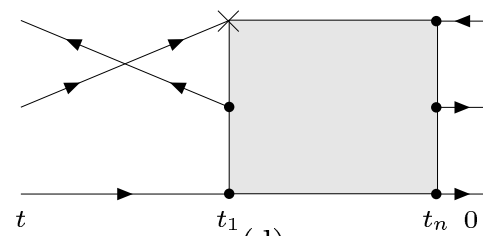

(d)

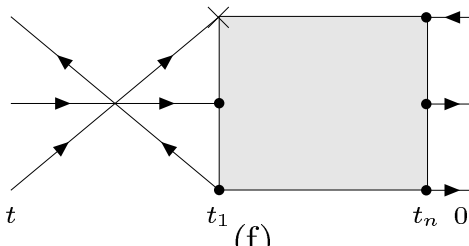

(f)

Figure 2: Diagrammatic representation of the contributions of order $n$ to the CLA (Equation (2.24)). The fictitious $\times$ interaction vertex represents a $R^{ \pm}$function as explained in the example (2.13).

This result can be easily verified by expanding with Wick's theorem, and then applying Equations (2.10) although the proof is somewhat lengthy. In Equation (2.19), the propagation between times $t$ and $t_{1}$ is described by the factor

$$
G_{0}\left(\alpha \beta \underline{\gamma}, \underline{\xi} \tau \rho ; t-t_{1}\right)=R^{-}\left(\gamma, \xi ; t-t_{1}\right) S_{0}^{h}\left(\beta, \tau ; t-t_{1}\right) S_{0}^{h}\left(\alpha, \rho ; t-t_{1}\right),
$$

this is similar to a non-interacting three-body propagator, except that the $S^{e}$ has been replaced by a $R^{-}$; as a shorthand notation we underline the electron indices that correspond to a $R^{-}$ factor. In a similar way $H_{U}\left(t_{1}\right)$ can give other possible ladder contributions to Equation (2.17); these come from the interaction of the $\gamma$ electron with one of the two holes; in this case is the other hole that goes to a $\times$ vertex and we meet the factors

$$
\begin{aligned}
& G_{0}\left(\alpha \underline{\beta} \gamma, \gamma^{\prime} \underline{\beta}^{\prime} \alpha^{\prime} ; t-t_{1}\right)=S_{0}^{e}\left(\gamma, \gamma^{\prime} ; t-t_{1}\right) R^{+}\left(\beta, \beta^{\prime} ; t-t_{1}\right) S_{0}^{h}\left(\alpha, \alpha^{\prime} ; t-t_{1}\right), \\
& G_{0}\left(\underline{\alpha} \beta \gamma, \gamma^{\prime} \beta^{\prime} \underline{\alpha^{\prime}} ; t-t_{1}\right)=S_{0}^{e}\left(\gamma, \gamma^{\prime} ; t-t_{1}\right) S_{0}^{h}\left(\beta, \beta^{\prime} ; t-t_{1}\right) R^{+}\left(\alpha, \alpha^{\prime} ; t-t_{1}\right) ;
\end{aligned}
$$

where underline hole indices correspond to a $R^{+}$contraction.

A glance to Equation (2.17) reveals that the last factor in the rhs of Equation (2.19) is the matrix element which enters $G_{n-1}$; hence we obtain the CLA form for one of the term of order $n$ of the development, corresponding to the diagram $(a)$ of Figure (2)

$$
-\sum_{\mu<\nu, \rho<\tau} U_{\mu \nu \rho \tau}\left[\sum_{\xi} \int_{-\infty}^{\infty} d t_{1} G_{0}\left(\alpha \beta \underline{\gamma}, \underline{\xi} \tau \rho ; t-t_{1}\right) G_{n-1}\left(\mu \nu \xi, \gamma^{\prime} \beta^{\prime} \alpha^{\prime} ; t_{1}\right)\right] .
$$


In other terms of the expansion, $H_{U}\left(t_{1}\right)$ describes the interaction of the $\gamma$ electron with the $\alpha$ and $\beta$ holes; in addition, each contribution has an exchange counterpart, obtained by crossing the hole lines going from $t$ to $t_{1}$. Thus, applying the CA to the other contractions of eq.(2.17) we get the six contributions represented in the fig.(2). Letting $n \rightarrow \infty$ and Fourier

transforming we obtain a linear system of equations for the CLA form $G^{C L A}\left(\alpha \beta \gamma, \gamma^{\prime} \beta^{\prime} \alpha^{\prime} ; \omega\right)$ of the three-body Green's function, namely

$$
\begin{gathered}
G^{C L A}\left(\alpha \beta \gamma, \gamma^{\prime} \beta^{\prime} \alpha^{\prime} ; \omega\right)=B_{0}\left(\alpha \beta \gamma, \gamma^{\prime} \beta^{\prime} \alpha^{\prime} ; \omega\right)- \\
\sum_{\xi}\left\{\sum_{\mu<\nu}\left[\sum_{\rho<\tau} U_{\mu \nu \rho \tau} B_{0}(\alpha \beta \underline{\gamma}, \underline{\xi} \tau \rho ; \omega)\right] G^{C L A}\left(\mu \nu \xi, \gamma^{\prime} \beta^{\prime} \alpha^{\prime} ; \omega\right)-\right. \\
\sum_{\mu<\nu, \rho<\tau} U_{\mu \nu \rho \tau}\left[B_{0}(\alpha \underline{\beta} \gamma, \nu \underline{\xi} \rho ; \omega) G^{C L A}\left(\mu \xi \tau, \gamma^{\prime} \beta^{\prime} \alpha^{\prime} ; \omega\right)+\right. \\
\left.\left.B_{0}(\underline{\alpha} \beta \gamma, \mu \tau \underline{\xi} ; \omega) G^{C L A}\left(\xi \nu \rho, \gamma^{\prime} \beta^{\prime} \alpha^{\prime} ; \omega\right)\right]\right\} .
\end{gathered}
$$

where the $B_{0}$ functions are

$$
\begin{gathered}
B_{0}\left(\alpha \beta \gamma, \gamma^{\prime} \beta^{\prime} \alpha^{\prime} ; \omega\right)=G_{0}\left(\alpha \beta \gamma, \gamma^{\prime} \beta^{\prime} \alpha^{\prime} ; \omega\right)-G_{0}\left(\beta \alpha \gamma, \gamma^{\prime} \beta^{\prime} \alpha^{\prime} ; \omega\right), \\
B_{0}(\alpha \beta \underline{\gamma}, \underline{\xi} \rho ; \omega)=G_{0}(\alpha \beta \underline{\gamma}, \underline{\xi} \tau \rho ; \omega)-G_{0}(\alpha \beta \underline{\gamma}, \underline{\xi} \rho \tau ; \omega), \\
B_{0}(\alpha \underline{\beta} \gamma, \nu \underline{\xi} \rho ; \omega)=G_{0}(\alpha \underline{\beta} \gamma, \nu \underline{\xi} \rho ; \omega)-G_{0}(\underline{\alpha} \beta \gamma, \nu \rho \underline{\xi} ; \omega), \\
B_{0}(\underline{\alpha} \beta \gamma, \mu \tau \underline{\xi} ; \omega)=G_{0}(\underline{\alpha} \beta \gamma, \mu \tau \underline{\xi} ; \omega)-G_{0}(\alpha \underline{\beta} \gamma, \mu \underline{\xi} \tau ; \omega) .
\end{gathered}
$$

The second, third and fourth lines of (2.24) come respectively from the (a,b), (c,d) and (e,f) diagrams of Figure (2); while the first two contributions come from the hole-hole interaction the others come from electron-hole interactions and convey information on the screening effects due to the electronic cloud which forms as a response to the deep electron ionization.

\subsection{Single-particle contribution}

At the same level of approximation we must consider the case when one (or both) the holes produced by the Auger transitions has the same spin as the screening electron. Consider the spin-diagonal components $G\left(\alpha \beta \gamma, \gamma^{\prime} \beta^{\prime} \alpha^{\prime} ; t\right)$, (with $\sigma_{\alpha^{\prime}}=\sigma_{\alpha}$ and so on); when the hole $\beta$ has the same z spin component as the electron, then, contracting $a_{\beta}^{\dagger}(t)$ with $a_{\gamma}(t)$ and $a_{\beta^{\prime}}$ with $a_{\gamma^{\prime}}^{\dagger}$ in (2.17) one obtains the extra contribution

$$
G^{s p}\left(\alpha \beta \gamma, \gamma^{\prime} \beta^{\prime} \alpha^{\prime} ; t\right)=(-1)\left\langle a_{\beta}^{\dagger} a_{\gamma}\right\rangle\left\langle a_{\gamma^{\prime}}^{\dagger} a_{\beta^{\prime}}\right\rangle S\left(\alpha, \alpha^{\prime} ; t\right)
$$

where $S\left(\alpha, \alpha^{\prime} ; t\right)$ stands for the time-ordered dressed one-body Green's function that can be expanded with

$$
S\left(\alpha, \alpha^{\prime} ; t\right)=\sum_{n}(-i)^{n} \int_{-\infty}^{\infty} d t_{1} \ldots \int_{-\infty}^{\infty} d t_{n}\left\langle T\left\{a_{\alpha}^{\dagger}(t) H_{U}\left(t_{1}\right) \ldots H_{U}\left(t_{n}\right) a_{\alpha^{\prime}}\right\}\right\rangle_{c} .
$$

and summed with the Dyson's equation [28] in terms of proper Self-Energy diagrams. 

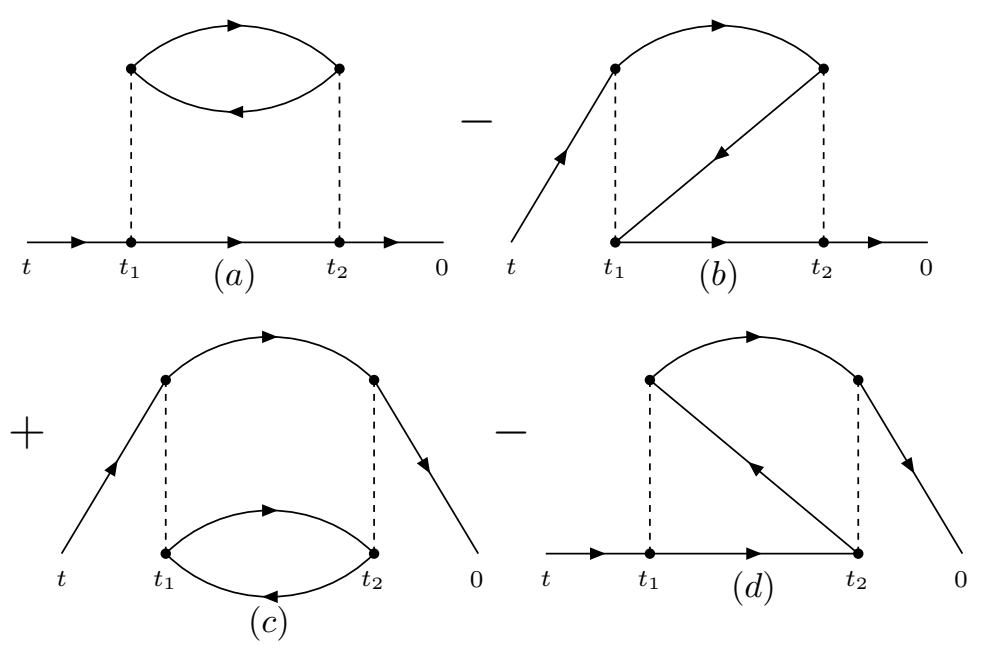

Figure 3: Topologically distinct contributions to the Second order self-energy. No tadpole diagrams occur because we expand in terms of Hartree-Fock spin-orbitals.

At second order of (2.27) we find eigth diagrams free of Hartree-Fock (tadpole) insertions that give rise to four topologically distinct ones as shown in Figure (3), which shows how the second-order self-energy can be expressed in terms of the hole-hole-electron Green's function $G$. The rules are: (i) write down a diagram for G; (ii) join the incoming electron line with one of the hole lines into an interaction vertex and draw an interaction line to the other hole; (iii) repeat the operation with the outgoing lines; (iv) do all that in all possible ways. This is a general result that can be extended to all orders yielding the Dyson form for the $S\left(\alpha, \alpha^{\prime} ; \omega\right)$

$$
\begin{aligned}
& S\left(\alpha, \alpha^{\prime} ; \omega\right)=S_{0}\left(\alpha, \alpha^{\prime} ; \omega\right)-\sum_{\mu^{\prime}<\nu^{\prime}, \rho^{\prime}<\tau^{\prime}} \sum_{\mu<\nu, \rho<\tau} U_{\mu \nu \rho \tau} U_{\mu^{\prime} \nu^{\prime} \rho^{\prime} \tau^{\prime}} \\
& \left\{\left[S_{0}(\alpha, \rho ; \omega) G\left(\mu \nu \tau, \nu^{\prime} \tau^{\prime} \rho^{\prime} ; \omega\right)-S_{0}(\alpha, \tau ; \omega) G\left(\mu \nu \rho, \nu^{\prime} \tau^{\prime} \rho^{\prime} ; \omega\right)\right] S\left(\mu^{\prime}, \alpha^{\prime} ; \omega\right)+\right. \\
& \left.\quad\left[S_{0}(\alpha, \tau ; \omega) G\left(\mu \nu \rho, \mu^{\prime} \tau^{\prime} \rho^{\prime} ; \omega\right)-S_{0}(\alpha, \rho ; \omega) G\left(\mu \nu \tau, \mu^{\prime} \tau^{\prime} \rho^{\prime} ; \omega\right)\right] S\left(\nu^{\prime}, \alpha^{\prime} ; \omega\right)\right\} ;
\end{aligned}
$$

at the second order this reduces to the four terms of Figure (3). We get a conserving approximation for the proper self-energy, using the CLA approximation for the three-body Green's functions of Equation (2.28).

By solving Dyson's equation (2.28) one can model XPS spectra from valence bands with low band filling; this is another field of application of our approach.

Having computed $S$, one finds how the Fermi energy $E_{F}$ is renormalised by the interaction. In general, we shall reference all the three-body spectra to the value of $E_{F}$ which are obtained by the corresponding one-body calculation. In the next Section, when comparing the line shapes with exact diagonalisation results, we shall align the Fermi levels accordingly.

The deep hole attracts a screening electron that can be directly involved in the Auger decay; this is the physical origin of the contribution (2.26) to the three-body Green's function. Locally, such processes leave the system with one hole in the final state. The presence of an one-body contribution in the Auger spectra from transition metals like $\mathrm{Ti}$ or Sc has been pointed out 
already [19, 21]. Besides the three-body (2.24) and one-body (2.26) diagrams, there are mixed contributions. Physically they represent interference contributions in which the system evolves from one-hole states to two-hole one electron states and back. Here we are going to neglect those diagrams for the sake of simplicity. Such terms can only be significant if the interaction is strong enough; however for strong interaction the one and three-body contributions are widely separated in frequency, so we may argue that the interference terms are not very important in general. Thus, we write

$$
G\left(\alpha \beta \gamma, \gamma^{\prime} \beta^{\prime} \alpha^{\prime} ; \omega\right)=G^{C L A}\left(\alpha \beta \gamma, \gamma^{\prime} \beta^{\prime} \alpha^{\prime} ; \omega\right)+G^{s p}\left(\alpha \beta \gamma, \gamma^{\prime} \beta^{\prime} \alpha^{\prime} ; \omega\right)
$$

\section{Comparison with exact diagonalisation results}

In this Section, we wish to test the CLA results against those of a model system that can be diagonalised exactly.

\subsection{Model Cluster}

We consider a 5 atom cluster, with 2 levels for each atom; the one-body basis elements are $\mid$ si $\sigma\rangle$ with $s=1, \ldots, 5$ the site index, $i=1,2$ the level index and $\sigma=\uparrow, \downarrow$ for the spin direction; the one-body energies are denoted by $\epsilon_{s j}$. The atoms $1 \ldots 4$ occupy the vertices of a square, and the Auger atom is at site 5 above the centre. Although this is just a model, we wish to use it to outline a possible procedure for the analysis of actual experimental data. Suppose we know a simple one-body Hamiltonian $H_{0}$ of the system, of the same level of sophistication as a tight-binding model of a solid, with a nearest neighbor hopping term:

$$
H_{0}=\sum_{s i \sigma} n_{s i \sigma} \epsilon_{s i}+\sum_{i j \sigma} \sum_{\left\langle s s^{\prime}\right\rangle} T_{i j}^{s s^{\prime}} a_{s i \sigma}^{\dagger} a_{s^{\prime} j \sigma},
$$

with real $T_{i j}^{s s^{\prime}}$. For nearest neighbor sites we assume identical matrices

$$
\left\{\mathbf{T}_{i j}\right\}=\left(\begin{array}{ll}
t_{11} & t_{12} \\
t_{12} & t_{11}
\end{array}\right)
$$

Next, suppose we have an estimate of the Hubbard $U$ and we wish to model the interactions by

$$
H_{H u b}=U\left[\sum_{s i j} n_{s i \uparrow} n_{s j \downarrow}+\sum_{s \sigma} n_{s 1 \sigma} n_{s 2 \sigma}\right] .
$$

The full interacting model Hamiltonian cannot be taken to be the sum of $H_{0}$ and $H_{H u b}$, because $H_{0}$ must contain the effects of the interactions on the one-body states, roughly, at the HartreeFock level. A mean field average of the interaction must be subtracted out. Therefore, we assume the model Hamiltonian

$$
H=H_{0}+H_{1} \text {, }
$$


where

$$
H_{1}=H_{H u b}-V_{H-F}
$$

and

$$
\begin{array}{r}
V_{H-F}=U\left\{\sum_{s i j}\left[\left\langle n_{s i \uparrow}\right\rangle n_{s j \downarrow}+n_{s i \uparrow}\left\langle n_{s j \downarrow}\right\rangle\right]+\sum_{s \sigma}\left[\left\langle n_{s 1 \sigma}\right\rangle n_{s 2 \sigma}+n_{s 1 \sigma}\left\langle n_{s 2 \sigma}\right\rangle+\right.\right. \\
\left.\left.\left(a_{s 1 \sigma}^{\dagger} a_{s 2 \sigma}\left\langle a_{s 1 \sigma} a_{s 2 \sigma}^{\dagger}\right\rangle+\text { h.c. }\right)\right]\right\} .
\end{array}
$$

where mean values are taken over the non-interacting ground state. In principle, if $H_{0}$ were a Self-Consistent-Field Hamiltonian, a self-consistent calculation of $\epsilon$ parameters and mean occupation numbers would be a more precise procedure. In this way, the Hartree-Fock contribution to the self-energy would be automatically enbodied in the bare propagators. In the present paper, we use non-interacting ground state averages, which is a cruder approximation, because we wish to privilege the simplicity of the procedure; the simplified approach already gives gratifying results (see below).

\subsection{Exact diagonalisation calculations}

We have chosen the following parameters in $\mathrm{eV}: \epsilon_{s 1}=0$ and $\epsilon_{s 2}=1$ for all sites; $t_{11}=1.4$ and $t_{12}=0.4$. In this way the bandwidth $W$, that is the difference between the extreme eigenvalues of $H_{0}$, is $\approx 9.6 \mathrm{eV}$, a reasonable value for a transition metal. We want the densities $D$ which are related to the positive time part of the Green's functions (1.1), and we are particularly interested in the diagonal elements $D\left(5 i \sigma_{i}, 5 j \sigma_{j}, 5 k \sigma_{k} ; \omega\right)$; these are usually the largest and must be non-negative.

These are given by (dropping the site index $s=5$ )

$$
D\left(i \sigma_{i}, j \sigma_{j}, k \sigma_{k} ; \omega\right)=\sum_{n}\left|\left\langle\psi\left|a_{i \sigma_{i}}^{\dagger} a_{j \sigma_{j}}^{\dagger} a_{k \sigma_{k}}\right| E_{N-1}^{(n)}\right\rangle\right|^{2} \delta\left(\omega+E_{N-1}^{(n)}-E\right),
$$

where $\mathrm{N}$ is the total number of electrons in the cluster, $|\psi\rangle$ is the interacting ground state with energy $\mathrm{E} ;\left|E_{N-1}^{(n)}\right\rangle$ is the complete set of interacting eigenstates with $(N-1)$ electrons. In one set of calculations we start from a ground state with $N=18$ valence electrons, since the maximum occupation in the cluster is 20 , we have two holes. We assume a non-magnetic ground state, with one hole for each spin; the configurations are $\left(\begin{array}{c}10 \\ 2\end{array}\right)^{2}=100$. In the final state, with two Auger holes of opposite spin and a spin-up electron we find $\left(\begin{array}{c}10 \\ 2\end{array}\right)\left(\begin{array}{c}10 \\ 1\end{array}\right)=450$ intermediate states with $(N-1)$ electrons.

In another set of calculations we start from a ground state with $N=16$, that is, two holes for each spin, and get $|\psi\rangle$ and $E$ by diagonalising a $\left(\begin{array}{c}10 \\ 2\end{array}\right)^{2} \times\left(\begin{array}{c}10 \\ 2\end{array}\right)^{2}=2025 \times 2025$ matrix, while for $\left|E_{N-1}^{n}\right\rangle$ we meet the maximum size of matrices in our calculations, namely $\left(\begin{array}{c}10 \\ 2\end{array}\right)\left(\begin{array}{c}10 \\ 3\end{array}\right)=5400$.

The site $s=5$ was chosen to be the Auger atom because in this way the problem is highly symmetric, and we can classify all the states according to the Irreducible Representations $(I R)$ 
of the $C_{4 v}$ Group, the Group of the square. Carring out in Equation (3.7) the summation on the $I R$ of the group we obtains

$$
D\left(i \sigma_{i}, j \sigma_{j}, k \sigma_{k} ; \omega\right)=\sum_{I R} \sum_{n}\left|\left\langle\psi\left|a_{i \sigma_{i}}^{\dagger} a_{j \sigma_{j}}^{\dagger} a_{k \sigma_{k}}\right| E_{N-1}^{(I R, n)}\right\rangle\right|^{2} \delta\left(\omega+E_{N-1}^{(I R, n)}-E\right)
$$

but the operators $a_{i \sigma}$ belong to the totalsymmetric representation $A_{1}$ of and so $|\psi\rangle$ and $\left|E_{N-1}^{n}\right\rangle$ must belong to the same IR. Since the ground state $|\psi\rangle$ for $N=16$ and $N=18$ is totalsymmetric we could restrict the summation to the $A_{1}$ sector.

$$
D\left(i \sigma_{i}, j \sigma_{j}, k \sigma_{k} ; \omega\right)=\sum_{n}\left|\left\langle\psi\left|a_{i \sigma_{i}}^{\dagger} a_{j \sigma_{j}}^{\dagger} a_{k \sigma_{k}}\right| E_{N-1}^{\left(A_{1}, n\right)}\right\rangle\right|^{2} \delta\left(\omega+E_{N-1}^{\left(A_{1}, n\right)}-E\right) .
$$

with a strong reduction of computing time.

\subsection{Comparison and evaluation of the CLA}

By the cluster calculations we want to study the physical consequences of the hole-electron interaction in the three-body density of states. Also, we wish to estimate the degree of validity of the CLA. We performed the comparison with the exact results for several values of $U / W$. By considering various possible electron populations $N \leq 20$ in the cluster we also varied the mean occupation of the Auger site in the non-interacting ground state

$$
\langle n\rangle=\frac{1}{4} \sum_{\sigma}\left\langle n_{51 \sigma}+n_{52 \sigma}\right\rangle .
$$

The test becomes more severe when $\langle n\rangle$ is reduced towards half filling and $U / W$ is increased. Many different densities of states are obtained from the matrix elements of $G(\omega)$; in the context of our theory, the density

$$
D_{1 h}(\omega) \equiv\left\langle\psi\left|a_{51 \uparrow}^{\dagger} a_{52 \downarrow}^{\dagger} a_{51 \uparrow} \delta(\omega+H-E) a_{51 \uparrow}^{\dagger} a_{52 \downarrow} a_{51 \uparrow}\right| \psi\right\rangle .
$$

is of special interest because in the diagrammatic series the annichilation of the spin-up electron by the hole of the same spin is particularly strong so we may expect that the one-body term is important. By contrast, in the density

$$
D_{2 h 1 e}(\omega) \equiv\left\langle\psi\left|a_{51 \uparrow}^{\dagger} a_{51 \downarrow}^{\dagger} a_{52 \uparrow} \delta(\omega+H-E) a_{52 \uparrow}^{\dagger} a_{51 \downarrow} a_{51 \uparrow}\right| \psi\right\rangle .
$$

the same contribution should be smaller and possibly absent. We shall consider these two examples in turn. In Appendix 9 we detail the application of the CLA to the problem at hand. Although the analytic development can be somewhat boring, the maximum size of the matrices involved is just 8 .

The left frame of Figure (4) shows $D_{1 h}$ for $U / W=0.25$ with $N=18$, which yields a population $\langle n\rangle=0.86$ on the Auger site. The density is dominated by a single peak at binding energy $\approx 4 \mathrm{eV}$, but also shows a pair of wings. Since correlation effects are moderate and the filling is fairly high, the CLA is still in good agreement with the exact results. 

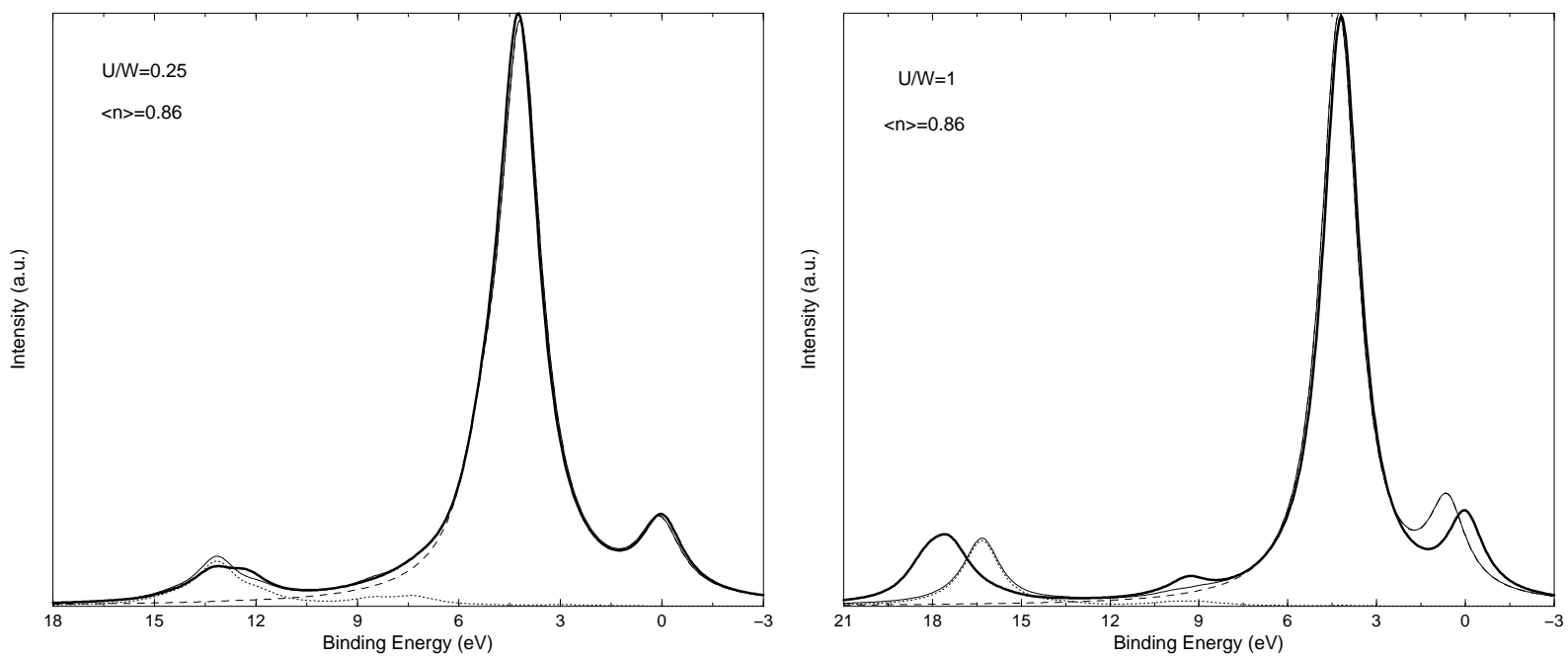

Figure 4: Binding energy dependence of $D_{1 h}$ for $\langle n\rangle=0.86$ with $U / W=0.25$ (left frame) and $U / W=1$ (right frame). Heavy line: exact result; light: CLA; dashed: 1-body contribution to the CLA result; dotted: 3-body contribution to the CLA result. The line shapes have been convolved with a Lorentzian $(\mathrm{FWHM}=0.75 \mathrm{eV})$.

In the right frame of Figure (国) the interaction is increased to $U / W=1$. The pattern does not show any major changes, except a shift and an increase of the structure at high binding energy. The shift $(\approx 5 \mathrm{eV})$ is large, which is understandable because the filling is high and the screening uneffective. The value of $U$ is well outside the scope of weak coupling approaches but the CLA still reproduces the exact results rather well.

Both the line shapes and the accuracy of the CLA are sensitive to $\langle n\rangle$. In a series of calculations, we set $N=16$, which yields $\langle n\rangle=0.72$.
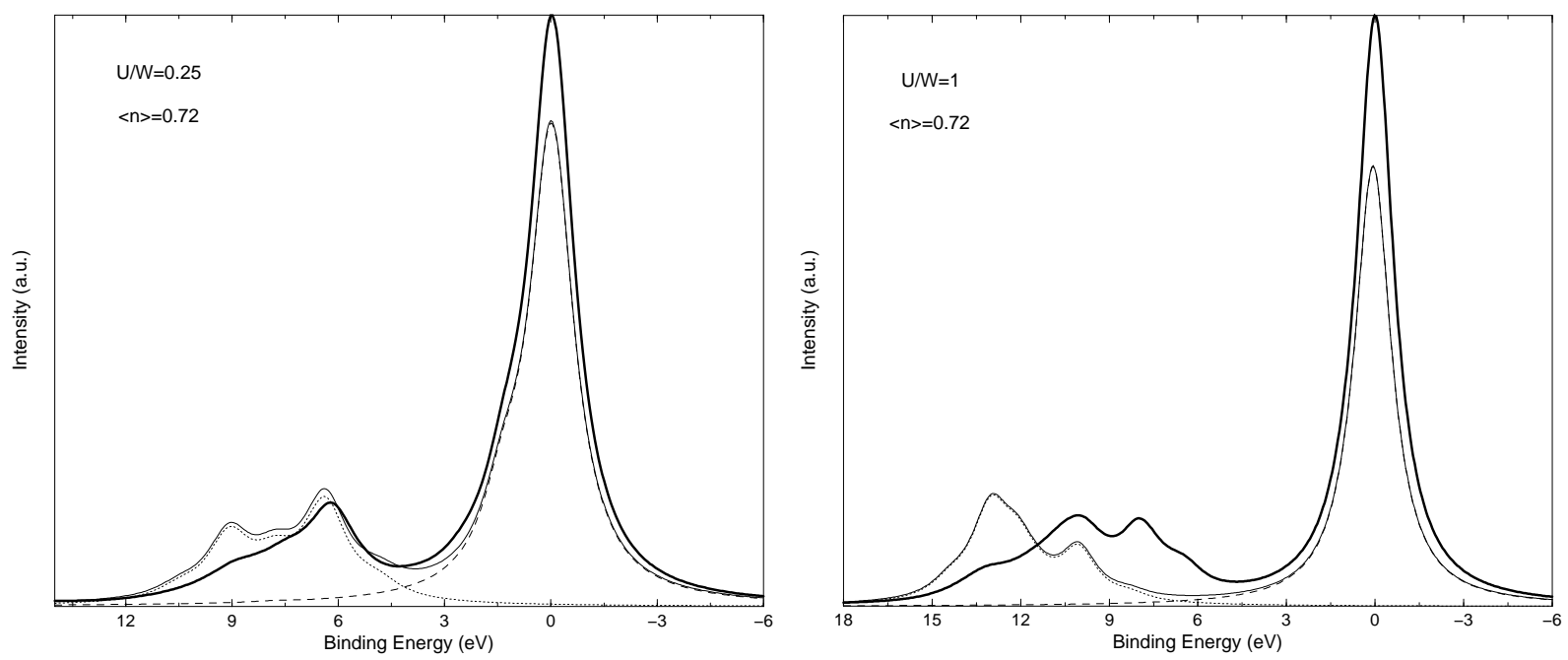

Figure 5: Binding energy dependence of $D_{1 h}$ for $\langle n\rangle=0.72$ with $U / W=0.25$ (left frame) and $U / W=1$ (right frame). Heavy line: exact result; light: CLA; dashed: 1-body contribution to the CLA result; dotted: 3-body contribution to the CLA result. The line shapes have been convolved with a Lorentzian (FWHM=0.75 eV). 

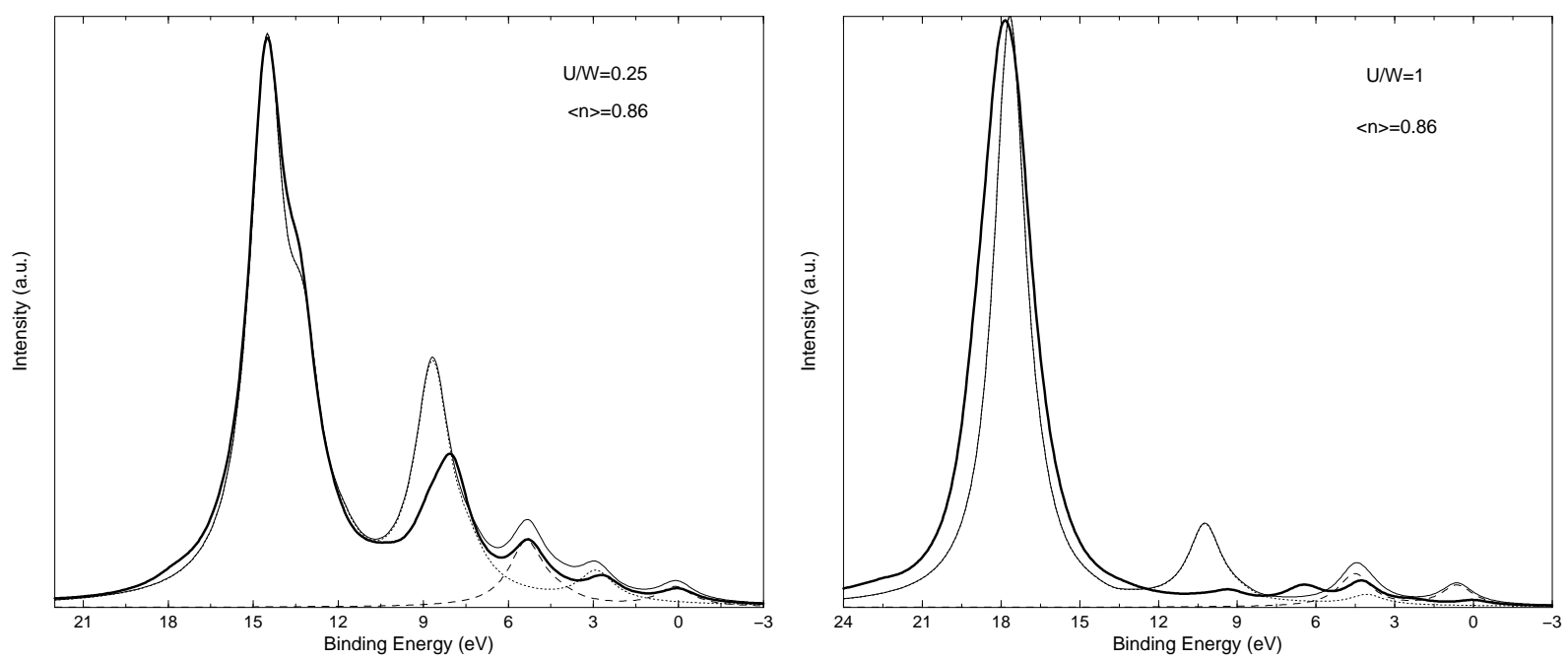

Figure 6: Binding energy dependence of $D_{2 h 1 e}$ for $\langle n\rangle=0.86$ with $U / W=0.25$ (left frame) and $U / W=1$ (right frame). Heavy line: exact result; light: CLA; dashed: 1-body contribution to the CLA result; dotted: 3-body contribution to the CLA result. The line shapes have been convolved with a Lorentzian $(\mathrm{FWHM}=0.75 \mathrm{eV})$.

In the left frame of Figure (51) a moderate interaction $U / W=0.25$ is assumed, and the exact results show a threshold peak centered at the Fermi level accompanied by a rich structure between 5 and $11 \mathrm{eV}$. The CLA underestimates somewhat the intensity of the main peak and does not faithfully reproduce the shape of the high binding energy structure, however the position of the main peak is correct and the overall line shape is in fair agreement. Moreover, the performance of the CLA does not break down quickly with increasing $U$ as weak-coupling approaches tend to do, but remains fairly stable. This can be seen in the right frame of Figure (5), where the comparison is done with $U / W=1$. Due to the effective screening in this case, the increase of $U$ does not cause a dramatic shift of the high binding-energy structure; this is borne out by the CLA and we may still speak of semiquantitative agreement. The CLA also explains the increase of the relative weight of the 3-body contribution with reducing band filling.

As one could expect, $D_{2 h 1 e}(\omega)$ has much more weight at high binding energies than $D_{1 h}(\omega)$, as one can see in Figure (6); in the right frame we have chosen the moderate coupling case $U / W=0.25$ with $\langle n\rangle=0.86$, like in Figure (蛋).

The overall agreement of the CLA with the exact results is quite good for the main peak, and even the small structures at lower binding energy are reproduced in some detail. With increasing $U$, these structures are largely lost (right frame of Figure (6)); the CLA deteriorates in those regions where the intensity is low, but the overall line shape is still satisfactory.

The most remarkable feature is that the large increase of the interaction strenght produces a rather small increase of the binding energy of the dominant peak compared to the left frame Figure (6). This would not be understandable if the peak were a two-hole resonance.

Physically, the shift is refrained by the screening of the two holes by the electron, which also becomes more effective with increasing $U$. Our simple approximation correctly accounts for the screening effect and consequently predicts the position of the main peak quite accurately. It is not surprising that $D_{2 h 1 e}(\omega)$ is more difficult to approximate in detail than $D_{1 h}(\omega)$, since many more, highly excited final states are reached with three quasiparticles than with one. 

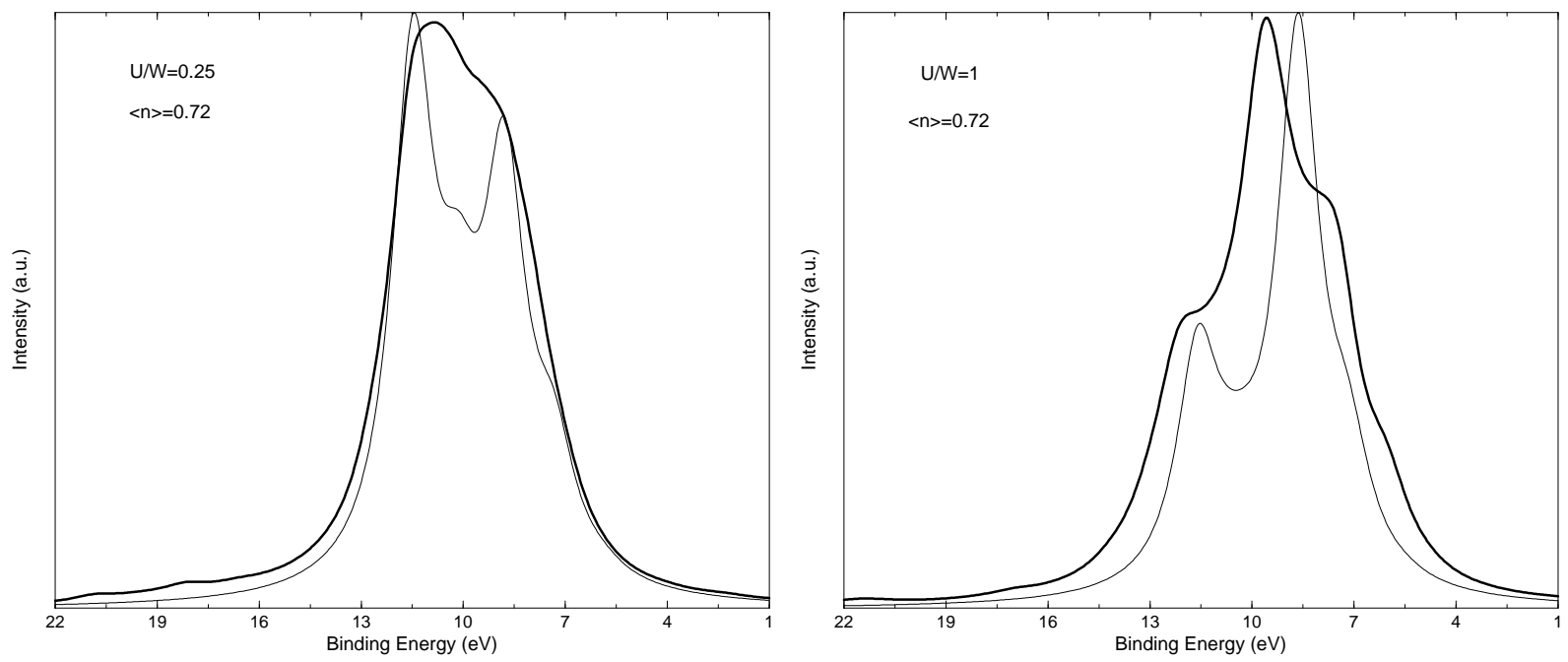

Figure 7: Binding energy dependence of $D_{2 h 1 e}$ for $\langle n\rangle=0.72$ with $U / W=0.25$ (left frame) and $U / W=1$ (right frame). Heavy line: exact result; light: CLA. The line shapes have been convolved with a Lorentzian $(\mathrm{FWHM}=0.75 \mathrm{eV})$.

A particularly hard case is shown in Figure (7) where the filling is rather low $(\langle n\rangle=0.72)$. No single-particle contribuition exists in this case because $\left\langle\psi\left|a_{51 \uparrow}^{\dagger} a_{52 \uparrow}\right| \psi\right\rangle=0$.

For $U / W=0.25$ (left frame) the agreement is fairly good. For $U / W=1$ (right frame) the exact line shape shows three partially resolved broad peaks covering a range of $\approx 10 \mathrm{eV}$ in binding energy. The CLA in such severe conditions misses the main peak position by $\approx 1$ $\mathrm{eV}$ and underevaluates the low binding energy shoulder; however, we can still claim at least a qualitative agreement with the results of the exact calculation.

In all cases we find that the Herglotz property is fully preserved; this is a most valuable feature which is not easily obtained for approximate three-body propagators. For instance, the approach of Ref. [21] fails in this respect at strong coupling.

Comparing the two frames of Figure (7) we observe the apparent negative- $U$ behaviour: increasing the interaction $\mathrm{U}$, the main peak shifts towards lower binding energies; this is a consequence of the interaction of the screening electron with the two Auger holes. We remark that a high enough $n_{h}$ is necessary to build up a localised screening cloud. This is why the negative- $U$ behavior is observed in the early transition metals, but not in the late ones.

\section{Conclusions}

To model Auger and APECS line shapes, we need a reliable practical recipe for a 3-body propagator, which poses much more difficult problems than the 1 and 2-particle Green's functions. The Core-Ladder-Approximation that we are proposing produces a dramatic simplification. The problem is reduced to the calculation of the mean-field one-body Green's function and to the diagonalisation of small matrices. Comparison with exact model results shows that one can capture the essential physics of a formidable problem which otherwise would require an excessive computational effort. 
Experience with the 1 and 2-body problems suggests that the 3-body ladder series, without self-energy and vertex corrections, is a prosiming approximation: it treats electrons and holes in the same way, and produces non-negative densities of states. From a mathematical viewpoint, the CLA that we are proposing is the first step of a procedure which eventually leads to the exact summation of the 3-body ladder series. Like perturbation theory and other approaches to the many-body problem, it allows systematic improvements, when required, at the cost of more computation. Physically, this simple approximation is well motivated and well balanced, and we provided evidence that it correctly describes the effect of the screening electron over the two final-state holes. The CLA is very accurate in the easy cases (high filling and/or small interaction); however, many transition metals are in the range of fillings and U where it is useful, and the approximation remains reasonable, Herglotz and qualitatively good even for $U / W=1$. This holds true for both the one-body features and the two-hole-one-electron contributions which result from the full 3-body Green's function. The property of the CLA of remaining qualitatively correct even at rather strong coupling, when all other simple approaches badly fail, is an appreciable feature. It depends on the fact that the Core Approximation becomes accurate at strong coupling, which is the most critical regime for perturbation theory; on the other hand, it allows to carry on the theory to all orders, while treating the screening electron and the Auger holes on equal footing. We are currently working out the application of the new approach to the line shape analysis of actual experimental spectra.

\section{A The Auger and APECS currents}

In a simplified form of the One-Step model [20] of the CVV Auger spectra, the current of electrons with energy $\epsilon_{k}$ measured in a Auger-Electron-Spectroscopy (AES) experiment is

$$
\mathcal{J}\left(\epsilon_{k}\right)=\int_{0}^{\infty} d t_{1} \int_{0}^{\infty} d t_{2} f\left(t_{1}, t_{2}\right) e^{i \epsilon_{k}\left(t_{1}-t_{2}\right)} .
$$

Here, letting $H_{S}$ represent the valence Hamiltonian without the core-hole, $|\psi\rangle$ its ground state, $H^{\prime}$ the valence Hamiltonian in the presence of the core-hole,

$$
f\left(t_{1}, t_{2}\right)=\sum_{m, m^{\prime}}\left\langle\psi\left|e^{i\left(H^{\prime}+i \Gamma\right) t_{2}}\right| m\right\rangle\left\langle m\left|H_{A}^{\dagger} e^{i H_{S}\left(t_{1}-t_{2}\right)} H_{A}\right| m^{\prime}\right\rangle\left\langle m^{\prime}\left|e^{-i\left(H^{\prime}-i \Gamma\right) t_{1}}\right| \psi\right\rangle,
$$

where

$$
H_{A}=\sum_{\alpha, \beta} M_{\alpha \beta} a_{\alpha} a_{\beta}
$$

produces the Auger holes with matrix elements $M$ in spin-orbitals denoted by Greek symbols; $\Gamma$ is an operator which produces virtual Auger transitions; core and free-electron operators have already been averaged out; the $m, m^{\prime}$ summations run over a complete set of valence states. In Ref. [21] a simple approximation was proposed. The basic idea was that the complete set of summations are largely exhausted by summing over just two orthogonal states, namely, $|\psi\rangle$ and the relaxed initial state of the Auger transition, $|\phi\rangle$, that is, the ground state of the valence electrons in the presence of the core-hole potential. In this scheme, the Auger spectrum 
has two main contributions, relaxed and unrelaxed. The unrelaxed contribution arises from $m=m^{\prime}=|\psi\rangle$, and can be expressed in terms of the two-hole Green's function $G_{\omega}^{(2)}$. The relaxed contribution arises from $m=m^{\prime}=|\phi\rangle$, and is proportional to

$$
\left\langle\phi\left|a_{\alpha}^{\dagger} a_{\beta}^{\dagger} e^{i H_{S}\left(t_{1}-t_{2}\right)} a_{\beta^{\prime}} a_{\alpha^{\prime}}\right| \phi\right\rangle .
$$

By a variational calculation Cini and Drchal [21] showed that

$$
|\phi\rangle \propto \sum_{\alpha_{l}} a_{\alpha_{l}}^{\dagger} a_{F}|\psi\rangle
$$

with $a_{\alpha_{l}}^{\dagger}$ creates an electron in a localized spin-orbital at the Auger site and $a_{F}$ annihilates an electron at the Fermi level. In this way the screening cloud is represented by a single electron that has moved from the Fermi surface to the empty local states of the Auger site. Using Equations (A.5) and (A.4) one obtains an expression for the relaxed contribution to the Auger line shape, involving the 3-body Green's function of Equation (11.1).

In APECS, on the other hand, one measures the Auger electron energy distribuition in concidence with the photoelectron energy, and one can selectively study the decay of the relaxed hole state. Semi-empirical studies [29] show that for a broad range of photoelectron kinetic energies the summation over $m, m^{\prime}$ is dominated by $|\phi\rangle$.

\section{B Proof of Equations (2.10) and (2.10')}

In first quantisation the operator $a_{\alpha}^{\dagger} a_{\beta}$ becomes $|\alpha\rangle\langle\beta|$, and one can easily show that $a_{\alpha}^{\dagger}(t) a_{\beta}\left(t^{\prime}\right)$ becomes $e^{i H_{0} t}|\alpha\rangle\langle\beta| e^{-i H_{0} t^{\prime}}$. The average of any one-body operator on a Slater determinant is the sum of the averages on the occupied spin-orbitals $k$. Therefore, letting $f_{k}=1$ for occupied states and $f_{k}=0$ for empty ones,

$$
\left\langle a_{\alpha}^{\dagger}(t) a_{\beta}\left(t^{\prime}\right)\right\rangle=\sum_{k} f_{k} e^{i \epsilon_{k}\left(t-t^{\prime}\right)}\langle k \mid \alpha\rangle\langle\beta \mid k\rangle .
$$

Therefore, summing over all the atomic spin-orbitals, one gets

$$
\sum_{\gamma}\left\langle a_{\alpha}^{\dagger}(t) a_{\gamma}\left(t^{\prime}\right)\right\rangle\left\langle a_{\gamma}^{\dagger}\left(t^{\prime}\right) a_{\beta}\right\rangle=\sum_{\gamma} \sum_{k, k^{\prime}} f_{k} f_{k^{\prime}} e^{i \epsilon_{k}\left(t-t^{\prime}\right)}\langle k \mid \alpha\rangle\langle\gamma \mid k\rangle e^{i \epsilon_{k^{\prime}} t^{\prime}}\left\langle k^{\prime} \mid \gamma\right\rangle\left\langle\beta \mid k^{\prime}\right\rangle .
$$

Now, exploiting the completeness of the $\gamma$ set, we obtain

$$
\sum_{\gamma}\left\langle a_{\alpha}^{\dagger}(t) a_{\gamma}\left(t^{\prime}\right)\right\rangle\left\langle a_{\gamma}^{\dagger}\left(t^{\prime}\right) a_{\beta}\right\rangle=\sum_{k} f_{k} e^{i \epsilon_{k} t}\langle k \mid \alpha\rangle\left\langle\beta \mid k^{\prime}\right\rangle=\left\langle a_{\alpha}^{\dagger}(t) a_{\beta}\right\rangle .
$$

In Equation (B.3) the times $t, t^{\prime}$ are arbitrary. We are free to insert $\theta$ functions to distinguish between positive and negative $t$ and ensure that $t^{\prime}$ is intermediate between $t$ and 0 . This proves Equations (2.10). 


\section{Application of the CLA}

From the diagonalisation of the one-body part of the problem, including the Hartree-Fock terms (3.6), one readily obtains the propagator $S_{0}^{h, e}(i \sigma, j \sigma ; t)$ where $i=1,2$ and $\sigma=\uparrow, \downarrow$. Since there are two local orbitals in this model and the matrix $\left\{\mathbf{T}_{i j}\right\}$ is real the set of equations $(2.16)$ represent a $3 \times 3$ linear problem that determines each component of the $R^{ \pm}$matrices, from which the $B_{0}$ matrices (2.25) can be easly obtained.

For clarity, we report the system which determines $R^{+}$:

$$
\begin{gathered}
(-i) S_{0}^{h}(1 \sigma, 1 \sigma ; t)=R^{+}(1 \sigma, 1 \sigma, t) S_{0}^{h}\left(1 \sigma, 1 \sigma ; 0^{+}\right)+R^{+}(1 \sigma, 2 \sigma, t) S_{0}^{h}\left(2 \sigma, 1 \sigma ; 0^{+}\right), \\
(-i) S_{0}^{h}(1 \sigma, 2 \sigma ; t)=R^{+}(1 \sigma, 1 \sigma, t) S_{0}^{h}\left(1 \sigma, 2 \sigma ; 0^{+}\right)+R^{+}(1 \sigma, 2 \sigma, t) S_{0}^{h}\left(2 \sigma, 2 \sigma ; 0^{+}\right), \\
(-i) S_{0}^{h}(2 \sigma, 2 \sigma ; t)=R^{+}(2 \sigma, 1 \sigma, t) S_{0}^{h}\left(1 \sigma, 2 \sigma ; 0^{+}\right)+R^{+}(2 \sigma, 2 \sigma, t) S_{0}^{h}\left(2 \sigma, 2 \sigma ; 0^{+}\right),
\end{gathered}
$$

yielding

$$
\left(\begin{array}{l}
R^{+}(1 \sigma, 1 \sigma, t) \\
R^{+}(1 \sigma, 2 \sigma, t) \\
R^{+}(2 \sigma, 2 \sigma, t)
\end{array}\right)=(-i)\left(\begin{array}{ccc}
\bar{n}_{11, \sigma} & \bar{n}_{12, \sigma} & 0 \\
\bar{n}_{12, \sigma} & \bar{n}_{22, \sigma} & 0 \\
0 & \bar{n}_{12, \sigma} & \bar{n}_{22, \sigma}
\end{array}\right)^{-1}\left(\begin{array}{c}
S_{0}^{h}(1 \sigma, 1 \sigma, t) \\
S_{0}^{h}(1 \sigma, 2 \sigma, t) \\
S_{0}^{h}(2 \sigma, 2 \sigma, t)
\end{array}\right)
$$

where $\bar{n}_{i j, \sigma} \equiv S_{0}^{h}\left(i \sigma, j \sigma ; 0^{+}\right)$. The diagonal $\bar{n}$ elements are much bigger than the off-diagonal, and the problem is well posed. A similar procedure gives the $R^{-}$functions using the propagators $S_{0}^{e}(i \sigma, j \sigma ; t)$.

We consider the following spin configurations:

$$
G\left(i j k, k^{\prime} j^{\prime} i^{\prime} ; \omega\right) \equiv G\left(i \uparrow j \downarrow k \uparrow, k^{\prime} \uparrow j^{\prime} \downarrow i^{\prime} \uparrow ; \omega\right)
$$

where the $i, j, i^{\prime}, j^{\prime}$ indices refer to the holes. To find them, we write down explicitly the CLA equations (2.24), where only the interactions involving the orbitals 1 and 2 at the Auger site $s=5$ appear. In the Hubbard model the interaction terms are proportional to the product of number operators, and this entails the condition

$$
U_{\mu \nu \rho \tau}=U \delta_{\mu, \rho} \delta_{\nu, \tau}
$$

That simplifies the set of equations (2.24) that reduce to a $(8 \times 8)$ linear system. It is convenient to use the shorthand notation where underlined numbers mark the position of $R^{ \pm}$functions, namely:

$$
\begin{aligned}
& G_{0}(i j \underline{k}, \underline{1} 11 ; \omega)=S_{0}^{h}(i, 1 ; \omega) S_{0}^{h}(j, 1 ; \omega) R^{-}(k, 1 ; \omega), \\
& G_{0}(i \underline{j} k, 1 \underline{1} 1 ; \omega)=S_{0}^{h}(i, 1 ; \omega) R^{+}(j, 1 ; \omega) S_{0}^{e}(k, 1 ; \omega), \\
& G_{0}(\underline{i j k}, 11 \underline{1} ; \omega)=R^{+}(i, 1 ; \omega) S_{0}^{h}(j, 1 ; \omega) S_{0}^{e}(k, 1 ; \omega),
\end{aligned}
$$


then, we may write the system in the form

$$
\begin{aligned}
& G_{0}\left(i j k, k^{\prime} j^{\prime} i ; \omega\right)-G^{C L A}\left(i j k, k^{\prime} j^{\prime} i ; \omega\right)= \\
& U\left\{\left[G_{0}(i j \underline{k}, \underline{111} ; \omega)-G_{0}(\underline{i} j k, 11 \underline{1} ; \omega)\right] G\left(111, k^{\prime} j^{\prime} i^{\prime} ; \omega\right)+\right. \\
& {\left[G_{0}(i \underline{k}, \underline{2} 11 ; \omega)-G_{0}(i \underline{j} k, 2 \underline{1} 1 ; \omega)-G_{0}(\underline{i j k}, 21 \underline{1} ; \omega)\right] G\left(112, k^{\prime} j^{\prime} i^{\prime} ; \omega\right)+} \\
& {\left[G_{0}(i j \underline{k}, \underline{1} 21 ; \omega)-G_{0}(\underline{i j k}, 12 \underline{1} ; \omega)\right] G\left(121, k^{\prime} j^{\prime} i^{\prime} ; \omega\right)+} \\
& {\left[G_{0}(i j \underline{k}, \underline{2} 21 ; \omega)-G_{0}(i \underline{j} k, 2 \underline{2} 1 ; \omega)-G_{0}(\underline{i j k}, 22 \underline{1} ; \omega)\right] G\left(122, k^{\prime} j^{\prime} i^{\prime} ; \omega\right)+} \\
& {\left[G_{0}(i j \underline{k}, \underline{1} 12 ; \omega)-G_{0}(i \underline{j} k, 1 \underline{1} 2 ; \omega)-G_{0}(\underline{i j k}, 11 \underline{2} ; \omega)\right] G\left(211, k^{\prime} j^{\prime} i^{\prime} ; \omega\right)+} \\
& {\left[G_{0}(i j \underline{k}, \underline{2} 12 ; \omega)-G_{0}(\underline{i j k}, 21 \underline{2} ; \omega)\right] G\left(212, k^{\prime} j^{\prime} i^{\prime} ; \omega\right)+} \\
& {\left[G_{0}(i j \underline{k}, \underline{1} 22 ; \omega)-G_{0}(i \underline{j} k, 1 \underline{2} 2 ; \omega)-G_{0}(\underline{i j k}, 12 \underline{2} ; \omega)\right] G\left(221, k^{\prime} j^{\prime} i^{\prime} ; \omega\right)+} \\
& \left.\left[G_{0}(i j \underline{k}, \underline{2} 22 ; \omega)-G_{0}(\underline{i} j k, 22 \underline{2} ; \omega)\right] G_{0}\left(222, k^{\prime} j^{\prime} i^{\prime} ; \omega\right)\right\} \text {. }
\end{aligned}
$$

For the $G^{s p}$ contribution we use Equation (2.28) for the propagator $S\left(j \downarrow, j^{\prime} \downarrow ; \omega\right)$,

$$
S\left(j \downarrow, j^{\prime} \downarrow ; \omega\right)=S_{0}\left(j \downarrow, j^{\prime} \downarrow ; \omega\right)-U^{2} \sum_{k k^{\prime}} S_{0}(j \downarrow, k \downarrow ; \omega) \Sigma\left(k \downarrow, k^{\prime} \downarrow ; \omega\right) S\left(k^{\prime} \downarrow, j^{\prime} \downarrow ; \omega\right) .
$$

Finally, we obtain the proper self-energies $\Sigma\left(k \downarrow, k^{\prime} \downarrow ; \omega\right)$ from Equation (2.28), where we insert the CLA form of the three body Green's function determined above. We recall that the onebody contributions arise from the anihilation of the up-spin electron and hole; therefore, one is left with the propagator for a down-spin hole, which is diagonal in spin because there are no spin-flip interactions in our model. Taking into account Equation (C.4), one finds

$$
\begin{gathered}
\Sigma(1 \downarrow, 1 \downarrow ; \omega)=G^{C L A}(1 \uparrow 1 \downarrow 1 \uparrow, 1 \uparrow 1 \downarrow 1 \uparrow ; \omega)+G^{C L A}(2 \uparrow 1 \downarrow 2 \uparrow, 2 \uparrow 1 \downarrow 2 \uparrow ; \omega)+ \\
2 G^{C L A}(1 \uparrow 1 \downarrow 1 \uparrow, 2 \uparrow 1 \downarrow 2 \uparrow ; \omega)+G^{C L A}(1 \downarrow 2 \downarrow 2 \downarrow, 2 \downarrow 2 \downarrow 1 \downarrow ; \omega), \\
\Sigma(1 \downarrow, 2 \downarrow ; \omega)=G^{C L A}(1 \uparrow 1 \downarrow 1 \uparrow, 1 \uparrow 2 \downarrow 1 \uparrow ; \omega)+G^{C L A}(1 \uparrow 1 \downarrow 1 \uparrow, 2 \uparrow 2 \downarrow 2 \uparrow ; \omega)+ \\
G^{C L A}(2 \uparrow 1 \downarrow 2 \uparrow, 1 \uparrow 2 \downarrow 1 \uparrow ; \omega)+G^{C L A}(2 \uparrow 1 \downarrow 2 \uparrow, 2 \uparrow 2 \downarrow 2 \uparrow ; \omega)+ \\
G^{C L A}(1 \downarrow 2 \downarrow 2 \downarrow, 1 \downarrow 1 \downarrow 2 \downarrow ; \omega), \\
\Sigma(2 \downarrow, 2 \downarrow ; \omega)=G^{C L A}(1 \uparrow 2 \downarrow 1 \uparrow, 1 \uparrow 2 \downarrow 1 \uparrow ; \omega)+G^{C L A}(2 \uparrow 2 \downarrow 2 \uparrow, 2 \uparrow 2 \downarrow 2 \uparrow ; \omega)+ \\
2 G^{C L A}(1 \uparrow 2 \downarrow 1 \uparrow, 2 \uparrow 2 \downarrow 2 \uparrow ; \omega)+G^{C L A}(2 \downarrow 1 \downarrow 1 \downarrow, 1 \downarrow 1 \downarrow 2 \downarrow ; \omega), \\
\Sigma(2 \downarrow, 1 \downarrow ; \omega)=\Sigma(1 \downarrow, 2 \downarrow ; \omega) .
\end{gathered}
$$

\section{Acknowledgments}

This work has been supported by the Istituto Nazionale di Fisica della Materia. One of us (A.M.) has been supported by the INFM scholarship "Analisi teorica della Tecnica A.P.E.C.S.", prot. 1350 . 


\section{References}

[1] P. Weightman, Rep. Prog. Phys. 45, 753 (1982); J. C. Riviere, Atomic Energy Reserch Report AERE-R10384 (1982).

[2] M. Cini, Solid State Comm. 20, 605 (1977).

[3] M. Cini, Solid State Comm. 24, 681 (1977).

[4] G. A. Sawatzky, Phys. Rev. Lett. 39, 504 (1977).

[5] C. Verdozzi, M. Cini, J. F. McGilp, G. Mondio, D. Norman, J. A. Evans, A. D. Laine, P. S. Fowles, L. Duò and P. Weightman, Phys. Rev. B 43, 9550 (1991).

[6] R.J.Cole, C. Verdozzi, M. Cini and P. Weigthmam, Phys. Rev. B 49, 13329 (1994).

[7] C. Verdozzi, M. Cini, J. A. Evans, R. J. Cole, A. D. Laine, P. S. Fowles, L. Duò and P. Weightman, Europhvsics Letters 16 (8), 743 (1991); M. Cini and C. Verdozzi, Physica Scripta T 41, 67 (1992); C. Verdozzi and M. Cini, Phys. Rev. B 51, 7412 (1995).

[8] M. Cini, A. Pernaselci and E. Paparazzo, J. Electron Spectroscopy and Rel. Phenomena 72, 77 (1995); A. Pernaselci and M. Cini, J. Electron Spectroscopy and Rel. Phenomena 82, 79 (1996).

[9] M. Cini, Surf. Sci. 87, 483 (1979).

[10] S. Doniach and M. Šunjić, J. Phys. C: Solid State Phys. 3, 285 (1970).

[11] V. Galitzkii, Soviet Phys. JEPT 7, 104 (1958).

[12] M. Cini and C.Verdozzi, J. Phys. C: Condens. Matter 1, 7457 (1989).

[13] M. Cini, M. De Crescenzi, F. Patella, N. Motta, M. Sastry, F. Rochet, R. Pasquali, A. Balzarotti and C. Verdozzi, Phys. Rev. B 41, 5685 (1990).

[14] V. Drchal and J. Kudrnovsky, J. Phys. F: Metal Phys. 12, 2443 (1984).

[15] M. Cini and C. Verdozzi, Solid State Comm. 57, 657 (1986).

[16] J. E. Houston, J. W. Rogers, R. R. Rye, F. L. Hutson and D. Ramaker, Phys. Rev. B 34, 1215 (1986); M. Cini and A. D'Andrea, in Auger Spectroscopv and electronic structure, Springer Series in Surface Science, Vol. 18 Edited by K. Wandelt, G. Mondio and G. Cubiotti (Springer, Heidelberg, 1989).

[17] P. Hedegård and F.U. Hillebrecht, Phys. Rev. B 34, 3045 (1986).

[18] D. K. G. de Boer, C. Haas and G. A. Sawatzky, J. Phys. F: Metal Phys. 14, 2769 (1984).

[19] D. D. Sarma, S. R. Barman, S. Nimkar and H. R. Krishnamurthy, Phys. Scri. T 41, 184 (1992). 
[20] O.Gunnarsson and K.Schönhammer, Phys. Rev. B 22, 3710 (1980).

[21] M. Cini and V. Drchal, J. Phys. C: Condens. Matter 6, 8549 (1994); M. Cini and V. Drchal, J. Electron Spectroscopy and Rel. Phenomena 72, 151 (1995).

[22] H. W. Haak, G. A. Sawatzky and T. D. Thomas, Phys. Rev. Lett. 41, 1825 (1978).

[23] H. W. Haak, G. A. Sawatzky, L. Ungier, J. K. Gimziewsky and T. D. Thomas, Rev. Sci. Instrum. 55, 696 (1984).

[24] G. A. Sawatzky, Auger photoelectron coincidence spettroscopy, Treatise on material science and technology, Vol. 30 Accademic Press. New York.

[25] E. Jensen, R. A. Bartynski, S. L. Hulbert and E. D. Johnson, Rev. Sci. Instrum. 63, 3013 (1992).

[26] O. Gunnarsson and K. Schönhammer, Phys. Rev. B 26, 2765 (1982).

[27] G. A. Sawatzky and A. Lenselink, Phys. Rev. B 21, 1390 (1980).

[28] R. D. Mattuck, A guide to Feynman diagrams in the Many-Body problem, McGraw-Hill, New York (1976) Chapter 10.

[29] M. Cini and A. Marini, to be published. 